\title{
Eryurium!a $e$ vesign da Informação em ambientes virtuais de aprendizagem e seus impactos na prática docente do ensino superior: estudos na interface da Turma Virtual do SIGAA
}

The ergonomics and information design in virtual learning environments and its impacts on the University professors practices: interface studies on SIGAA's Virtual Class

\author{
André Grilo, Thiago Marques, Laura Fernandes, Gabriela Gentile
}

Ensino Superior, Tecnologias na Educação, Ergonomia Cognitiva, Design da Informação

Este trabalho investigou aspectos de ergonomia cognitiva no design de informação de interfaces gráficas, enfatizando a atividade de docentes do ensino superior. A pesquisa utilizou como objeto a Turma Virtual, módulo AVA disponibilizado pelo Sistema Integrado de Gestão de Atividades Acadêmicas (SIGAA), desenvolvido e mantido pela Universidade Federal do Rio Grande do Norte (UFRN). Participaram do estudo docentes oriundos de diversas unidades acadêmicas da Instituição, propiciando diferentes relatos de experiência e perspectivas quanto ao uso da ferramenta em seu cotidiano de ensino e gestão das disciplinas. A partir dos dados coletados, foram identificados os principais problemas informacionais em funcionalidades específicas da Turma Virtual, e elaborada uma proposta de redesign da interface, a partir de princípios ergonômicos de design da informação e dos relatos dos usuários. Uma demonstração do protótipo foi avaliada por um grupo heterogêneo de docentes por meio de questionários. Os resultados apontaram níveis positivos de satisfação dos usuários com a proposta apresentada, registrando sugestões de novos melhoramentos e evoluções na interface, bem como o interesse dos docentes participantes em utilizarem a nova interface em suas atividades acadêmicas.

\section{Higher Education, Technology in Education, Cognitive Ergonomics, Information Design}

\begin{abstract}
This paper discusses about an investigation on cognitive ergonomics aspects in the information design of graphical interfaces, focusing university professors's activities. The research object was the Virtual Class, virtual learning environment (VLE) of an academic system developed and maintained by the Federal University of Rio Grande do Norte, Brazil. The participants were professors from different locations of institution, which related about their experiences and perspectives about the VLE in their education contexts and disciplines management. From the data collected, it was identified the main interface's information problems and then was designed a new interface concept, through ergonomics and information design criteria and the experiences reported by the users. The prototype was evaluated by a heterogeneous group of potential users. The results point to the significant approval by the users, that suggested new improvements on interface, also they tell about their interest to use the new interface in their activities.
\end{abstract}

\section{Introdução}

As inovações em informática iniciadas no século $X X$ possibilitaram novas formas de obter conhecimento (Pereira, Schmitt \& Dias, 2007). Mais pessoas têm acessado informações de diferentes plataformas, e as redes sociais e outros espaços de conteúdo na internet alteraram a maneira de buscar informações e assimilar novos conhecimentos, ressignificando processos de aprendizagem (Cortella \& Dimenstein, 2015). Tais avanços tecnológicos e mudanças comportamentais catalisados pela web têm exigido dos docentes constante atualização e uso de recursos consoantes às realidades de cada nova turma ingressante nas universidades. Por outro lado, a web têm encurtado a relação espaço e tempo entre educadores e educandos, podendo servir como recurso para o ensino, tanto na forma como no conteúdo (Nunes \& Gonçalves, 2015). Desse fenômeno, surgiram soluções como os ambientes virtuais de aprendizagem (AVA), sobre os quais discorre este trabalho, de uma perspectiva da ergonomia cognitiva e do design da informação.

Anais do 8을 e 8 CONGIC

Guilherme Santa Rosa; Cristina Portugal (orgs.)

Sociedade Brasileira de Design da Informação - SBDI

Natal | Brasil | 2017

ISBN 978-85-212-1305-5
Proceedings of the $8^{\text {th }}$ CIDI and $8^{\text {th }}$ CONGIC

Guilherme Santa Rosa; Cristina Portugal (orgs.)

Sociedade Brasileira de Design da Informação - SBDI

Natal| Brazil | 2017

ISBN 978-85-212-1305-5 


\section{CIDI 2017

\subsection{Ambientes Virtuais de Aprendizagem}

Galafassi, Gluz \& Galafassi (2014:41) definem ambientes virtuais de aprendizagem como: "tecnologias integradoras e abrangentes capazes de organizar e definir um ponto focal para os processos educacionais mediados por computador, apoiar os professores e promover o engajamento dos alunos". Ainda segundo os autores, os recursos oferecidos pelos AVA encurtam a relação de distância e tempo entre alunos e professores, permitindo uma comunicação mais ágil entre os sujeitos. Os AVA contribuem de maneira substancial em abordagens educacionais mediadas por computador. Embora bastante evidenciados nas modalidades de ensino a distância $(E a D)$, muitas instituições têm adotado os AVA como uma ferramenta de ensino híbrido, servindo como extensão da sala de aula. Além do ambiente acadêmico ou escolar, organizações têm adotado os AVA como estratégia de educação corporativa (Pereira, Schmitt \& Dias, 2007).

Okada (2003:6) coloca que "Para compreender melhor ambientes virtuais colaborativos e cooperativos, é necessário não só refletir sobre a concepção de colaboração e cooperação, mas também, analisar as estruturas e interfaces contidas nestes ambientes". Além disso, segundo a autora, não é suficiente a instituição disponibilizar tais ambientes se a metodologia for conduzida nos moldes tradicionais. Esses novos espaços, então, requerem abordagens diferentes, o que traz implicações não apenas na metodologia de ensino, mas também na concepção de tais ambientes, a partir da reflexão sobre como suas configurações influenciam na experiência dos usuários. Tais questões transitam pelas dimensões ergonômica, interfacial e informacional presentes no design desses artefatos.

\subsection{Ergonomia e design da informação}

A ergonomia, termo que deriva da junção entre os termos gregos "ergon" e "nomon" (trabalho" e "regras", respectivamente), busca enxergar a atividade de trabalho como um sistema, caracterizado pela relação entre o homem, a tarefa e a máquina, sendo esta última qualquer dispositivo, seja um artefato físico, digital ou ambiental (lida, 2005). Segundo a Associação Brasileira de Ergonomia (ABERGO, 2008:1): "Os ergonomistas contribuem para o planejamento, projeto e a avaliação de tarefas, postos de trabalho, produtos, ambientes e sistemas de modo a torná-los compatíveis com as necessidades, habilidades e limitações das pessoas."

O estudo da ergonomia está dividido nos seguintes domínios (ABERGO, 2008): Ergonomia Física (aspectos mecânicos e biológicos), Ergonomia Cognitiva (aspectos psicológicos) e Ergonomia Organizacional (aspectos sociais e logísticos). É importante ressaltar que os três aspectos estão interligados, em maior ou menor grau, visto que as relações de trabalho se perfazem no espaço, nos artefatos físicos e, atualmente - em uma sociedade marcada pelo digital -, também nas relações informacionais das mídias eletrônicas. Na definição proposta pela ABERGO, o objetivo basilar da ergonomia está na busca pela compatibilidade entre os sistemas e os indivíduos que com eles interagem. Tal perspectiva vem ao encontro da visão de design proposta para o desenvolvimento de produtos, que, na visão de Norman (2010), devem ser desenvolvidos conforme a maneira que as pessoas se comportam, centrando a tecnologia nas pessoas.

O design da informação (DI), por sua vez, é o ramo do design gráfico (ou programação visual) cuja atividade está relacionada ao projeto da informação em suportes físicos ou digitais, no segmento editorial, publicitário ou na web, por exemplo. O design da informação está presente no cotidiano da sociedade, como em embalagens de produtos e nas sinalizações de ambientes internos ou externos, manuais, mapas e diagramas, dentre outros.

Historicamente, o campo de estudo do design da informação origina-se como ciência multidisciplinar, no final da década de 1970, com a publicação Information Design Journal, em 1979, organizada por designers gráficos que passaram a discutir sobre o tema à época. Na década seguinte, em 1986, é fundado o International Institute for Information Design (IIID), baseado na Áustria (IIID, 2017). Em 1991, é organizada a Information Design Association (IDA), unindo os conhecimentos de especialistas em design gráfico e em estudos da linguagem (IDA, 2014). No ano de 2002, foi organizada a Sociedade Brasileira de Design da Informação (SBDI), instituição sem fins lucrativos e de caráter científico (SBDI, 2016).

No design da informação, consideram-se os atributos de cada elemento da composição gráfica como formas de linguagem visual, que entregam a informação ao usuário. Assim, a composição de texto, a hierarquia e sequência de informações, a escolha da tipografia e das cores auxiliam a transmissão de mensagens em diferentes níveis de compreensão e assimilação visual (Samara, 2011; Spiekerman, 2011). Bertin (1967), um dos primeiros teóricos sobre programação visual e considerado um dos principais 


\section{CIDI 2017 告 cold

precursores do DI, propôs o conceito de variáveis visuais, considerando as diferentes formas e atributos com que elementos gráficos podem se apresentar em uma composição (Figura 1).

Figura 1: Variáveis visuais propostas por Jacques Bertin. (Elaboração dos autores, baseado em Bertin, 1967)

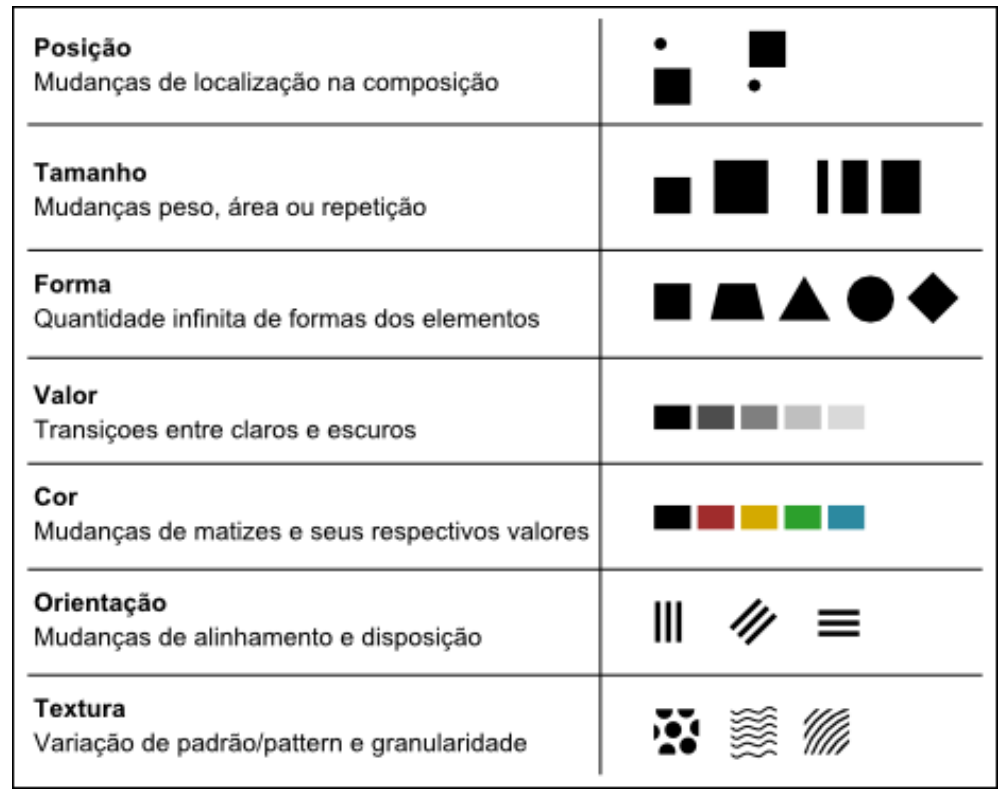

O modelo de análise de Mijksenaar (1997) propõe uma classificação das variáveis visuais originalmente propostas por Bertin (1967):

- Variáveis Diferenciadoras: cor, tipografia, ícone, ilustração, etc.;

- Variáveis Hierárquicas: posição e sequência da informação, peso da tipografia e estilo (e.g., negrito, itálico), alinhamento, etc.;

- Variáveis de Suporte: contornos, caixas, área de cor, sombreamento, etc.

As variáveis visuais influenciam a experiência informacional e devem ser consideradas durante a elaboração de composições gráficas, cabendo ao designer orientar o usuário na navegação e interação com as informações dispostas na interface (Morville \& Rosenfeld, 2007). Nesse sentido, o design da informação atua como parte integrante de interfaces ergonômicas ao usuário, uma vez que tais decisões de design podem comprometer ou não a tarefa de assimilação informacional dos sujeitos, devendo ser consideradas suas necessidades e capacidades.

A Information Design Association, em sua página oficial (IDA, 2014), destaca o seguinte questionamento: "Modern life is saturated with complex information and data. For information to have impact, it must be easy to find, simple to use, and instantly understandable. In short, information needs to be designed". De fato, a sociedade atual é baseada na informação e nas tecnologias que provém acesso para esta, e seu processamento e assimilação requerem um pensamento de projeto - design - dessa informação. Esse entendimento vai ao encontro das questões de pesquisa que motivam este trabalho:

- Como o design da informação em interfaces de ambientes virtuais de aprendizagem afeta a ergonomia do trabalho docente do ensino superior?

- Como oferecer tecnologias e sistemas cujas interfaces sejam informacionalmente adequadas para a prática docente?

Anais do $8^{\circ}$ CIDI e $8^{\circ}$ CONGIC

Guilherme Santa Rosa; Cristina Portugal (orgs.)

Sociedade Brasileira de Design da Informação - SBDI

Natal | Brasil | 2017

ISBN 978-85-212-1305-5
Proceedings of the $8^{\text {th }}$ CIDI and $8^{\text {th }}$ CONGIC

Guilherme Santa Rosa; Cristina Portugal (orgs.)

Sociedade Brasileira de Design da Informação - SBDI

Natal| Brazil | 2017

ISBN 978-85-212-1305-5 


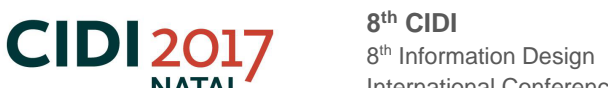 \\ $8^{\text {th }}$ CONGIC \\ $8^{\text {th }}$ Information Design

\section{Contexto da pesquisa}

Como objeto de estudo, foi analisada a interface gráfica da Turma Virtual (Figura 2), módulo AVA oferecido no Sistema Integrado de Gestão de Atividades Acadêmicas (SIGAA). O sistema foi desenvolvido, mantido e é adotado pela Universidade Federal do Rio Grande do Norte (UFRN). A população-alvo da investigação foram os docentes ativos da Instituição.

Figura 2: Captura de tela. Turma Virtual do SIGAA (Fonte: SINFO/UFRN)

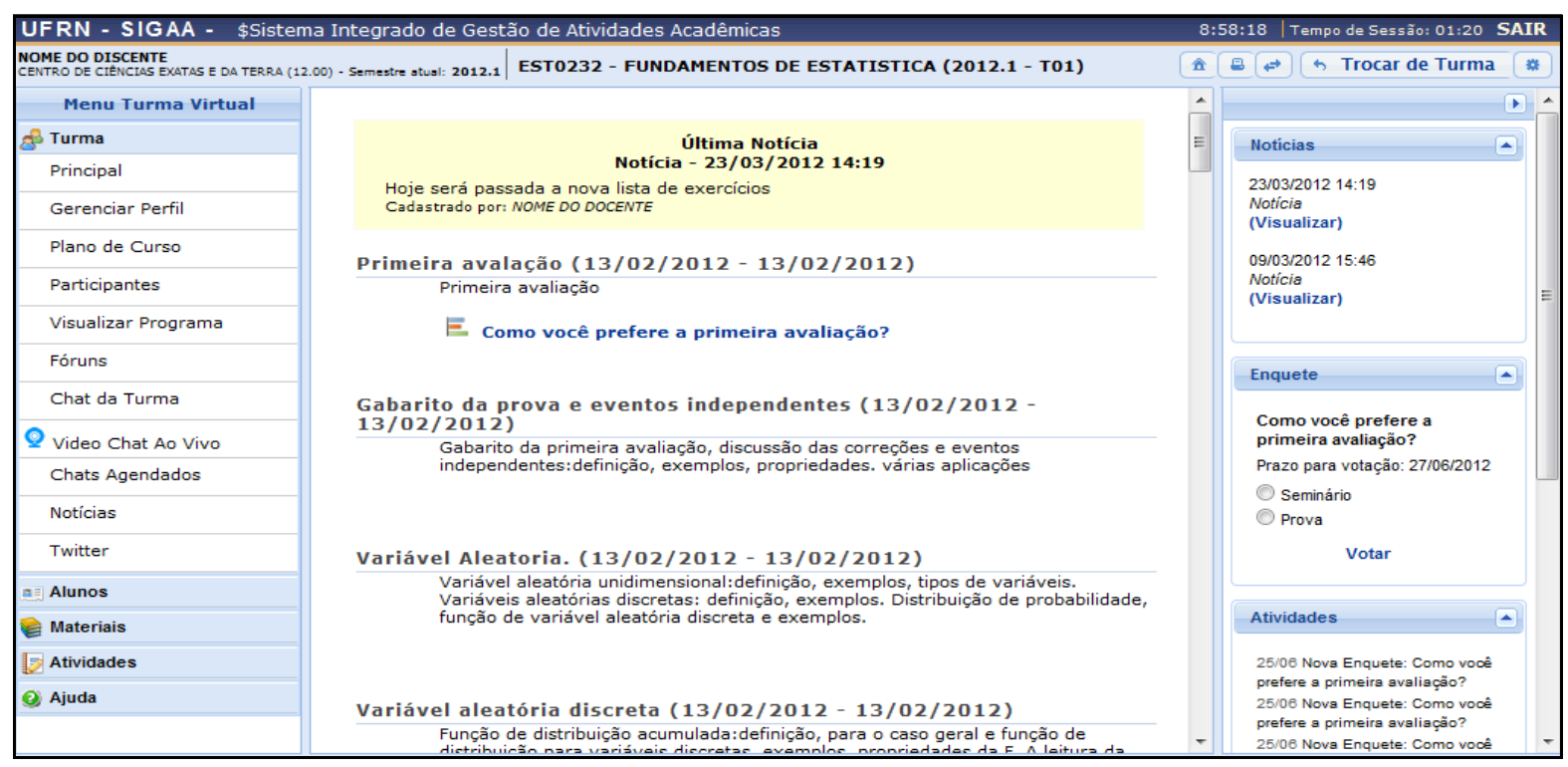

Em termos quantitativos, o SIGAA contabilizou, no ano de 2016, uma base de usuários ativos (discentes e docentes) de cerca de 40 mil usuários na Instituição (SINFO, 2016a). Somado a isso, a UFRN realiza termos de cooperação com outras instituições de ensino em nível nacional, contando com uma rede de cerca de 30 universidades que adotam os sistemas integrados, em variadas localidades das cinco regiões do território brasileiro (SINFO, 2016b). Nesse sentido, a Turma Virtual é um módulo de acentuado impacto na vida acadêmica de diversas instituições de ensino superior.

\subsection{Histórico}

Em estudo anterior (Grilo \& Dias, 2016), foi realizada uma pesquisa inicial por meio de questionários enviados eletronicamente. A pesquisa reuniu um grupo de 27 docentes da UFRN. Os participantes informaram a frequência de utilização de funcionalidades disponíveis na Turma Virtual (Tabela 1).

Tabela 1: Funcionalidades mais frequentes segundo os docentes (Fonte: Dados da pesquisa)

\begin{tabular}{lc}
\hline Funcionalidade & $\boldsymbol{f ( \% )}$ \\
\hline Cadastrar tópico de aula & $88 \%$ \\
\hline Cadastrar tarefa & $81,4 \%$ \\
\hline Cadastrar notícias & $92,5 \%$ \\
\hline Cadastrar frequência & $92,5 \%$ \\
\hline Cadastrar planos de curso & $59 \%$ \\
\hline Inserir arquivo na turma & $96,2 \%$ \\
\hline
\end{tabular}

Anais do 8 CIDI e 8 CONGIC

Guilherme Santa Rosa; Cristina Portugal (orgs.)

Sociedade Brasileira de Design da Informação - SBDI

Natal | Brasil | 2017

ISBN 978-85-212-1305-5

Proceedings of the $8^{\text {th }} \mathrm{CIDI}$ and $8^{\text {th }}$ CONGIC

Guilherme Santa Rosa; Cristina Portugal (orgs.)

Sociedade Brasileira de Design da Informação - SBDI

Natal| Brazil | 2017

ISBN 978-85-212-1305-5 


\section{CIDI 2017

Realizou-se também uma navegação exploratória na interface, em que foram detectadas não conformidades da interface com cinco heurísticas de usabilidade (Nielsen, 1995): Visibilidade e estado atual do sistema; Controle e liberdade ao usuário; Consistência e padrões; Auxiliar os usuários a reconhecer e reverter situações de erro; Reconhecimento e memorização. Durante a navegação exploratória, também foram identificados problemas de acessibilidade, como ruídos de comunicação para leitores de tela e de navegação via teclado, além da ausência de versões de alto-contraste para pessoas com baixa visão. Verificou-se ausência de recurso de busca. Um dos docentes participantes fez um importante questionamento quanto ao uso da Turma Virtual, afirmando que se esta não fosse prática e intuitiva para o docente, dificilmente seria adotada pelos alunos, que dependem da atualização desse ambiente para acessar arquivos, notas, notícias. Ou seja, quanto mais ergonômica a interface para o docente, mais propícia pode ser a adesão dos estudantes à ferramenta e à metodologia aplicada pelo educador.

\section{Procedimentos e técnicas}

Realizou-se intervenção na interface do objeto de pesquisa, parametrizando o redesign a partir do modelo teórico de análise de variáveis visuais, proposto por Mijksenaar (1997). Foi adotado um framework idealizado e aplicado em pesquisas anteriores na Instituição (Grilo, 2016; Grilo, 2017; Grilo, Silva \& Costa, 2017) para o gerenciamento visual das ideias geradas ao longo do processo de redesign, consistindo em reunir visualmente em um quadro o Diagnóstico dos problemas (D), Requisitos e restrições (R), Alternativas de solução (A) e os Feedbacks (F) das partes interessadas (usuários e equipe desenvolvedora). $O$ procedimento envolveu as seguintes técnicas:

- Elaboração de protótipo da nova interface, a partir dos problemas relatados pelos usuários;

- Apresentação de vídeo demonstrativo da nova interface para os docentes;

- Questionário de validação enviado aos docentes $(n=103)$.

\section{Resultados e discussões}

A Figura 3 contém os principais insights gerados colaborativamente durante o processo de análise e síntese do questionário da pesquisa anterior, bem como os primeiros feedbacks obtidos com usuários e desenvolvedores. A sequência de leitura dos quadrantes se descreve em sentido horário, começando pelo Diagnóstico (D).

Figura 3: Alguns dos insights gerados para proposta ergonômica de design da informação na interface (Elaboração dos autores)

Anais do 8 CIDI e 8 CONGIC

Guilherme Santa Rosa; Cristina Portugal (orgs.)

Sociedade Brasileira de Design da Informação - SBDI

Natal | Brasil | 2017

ISBN 978-85-212-1305-5
Proceedings of the $8^{\text {th }} \mathrm{CIDI}$ and $8^{\text {th }}$ CONGIC

Guilherme Santa Rosa; Cristina Portugal (orgs.)

Sociedade Brasileira de Design da Informação - SBDI

Natal| Brazil | 2017

ISBN 978-85-212-1305-5 


\section{CIDI2017 $8^{\mathrm{fn} C 1 D}$

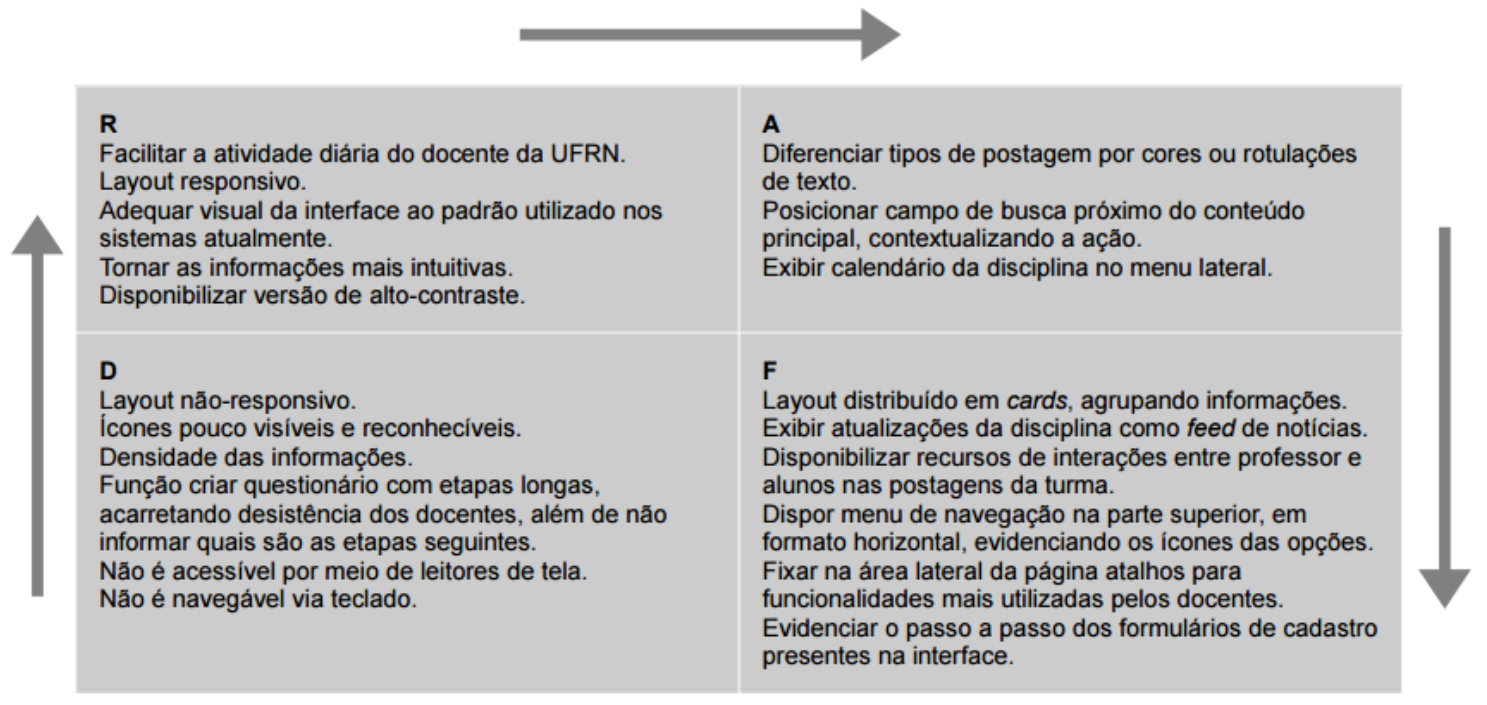

A partir dos insigths gerados, foi elaborada uma proposta de nova interface (Figura 4), a qual buscou contemplar os problemas ergonômicos descritos pelos usuários quanto às funcionalidades frequentes, bem como aspectos gerais da interface.

Figura 4: Redesign da interface. (Elaboração dos autores)

Anais do $8^{\circ}$ CIDI e 8 CONGIC

Guilherme Santa Rosa; Cristina Portugal (orgs.)

Sociedade Brasileira de Design da Informação - SBDI

Natal | Brasil | 2017

ISBN 978-85-212-1305-5
Proceedings of the $8^{\text {th }} \mathrm{CIDI}$ and $8^{\text {th }}$ CONGIC

Guilherme Santa Rosa; Cristina Portugal (orgs.)

Sociedade Brasileira de Design da Informação - SBDI

Natal| Brazil | 2017

ISBN 978-85-212-1305-5 


\section{CIDI 2017 intin

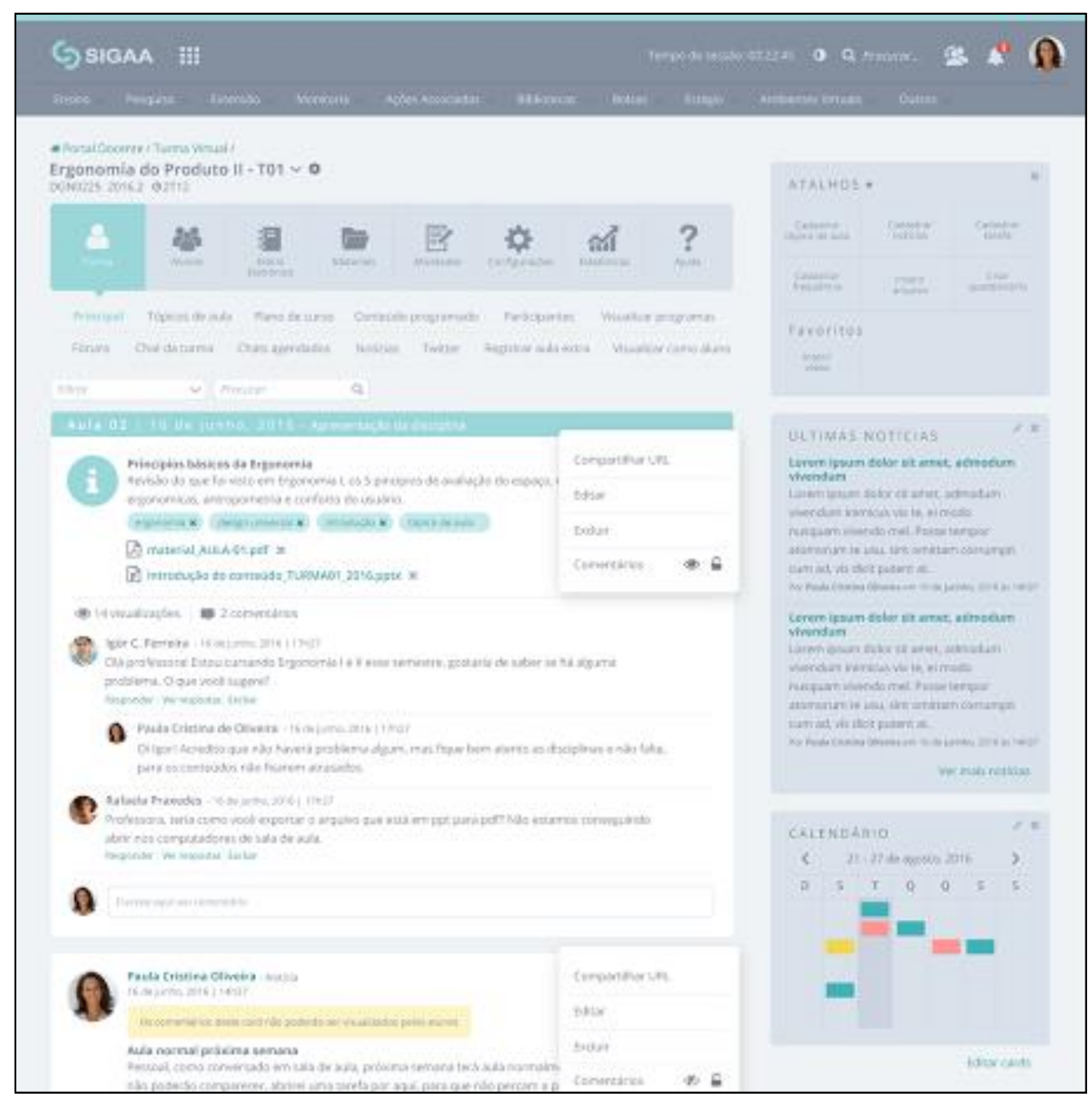

O redesign da interface baseou-se na nova identidade visual dos sistemas da instituição (Agecom, 2016). Propuseram-se mudanças tanto no aspecto de distribuição das informações, como também na apresentação destas, utilizando de diferentes variáveis visuais de suporte, diferenciadoras e hierárquicas. 0 menu é caracterizado pelo uso duas variáveis visuais diferenciadoras: as cores e os ícones, além da variável de suporte - a área do menu. Na solução proposta, o menu foi disposto na parte superior do layout, em formato horizontal, destacando os itens. Os subitens são exibidos com a sobreposição do cursor, em rótulos de texto, organizados de maneira que o usuário possa perceber qual caminho percorreu para chegar à seção atual (variável hierárquica). O professor pode alterar a disciplina clicando no ícone de seta posicionado à direita do nome da turma, que carregará um menu para selecionar outras disciplinas (Figura $5)$.

Figura 5: Menu de contexto para alternar visualização de disciplinas. (Elaboração dos autores)

Anais do 8 CIDI e 8 CONGIC

Guilherme Santa Rosa; Cristina Portugal (orgs.)

Sociedade Brasileira de Design da Informação - SBDI

Natal | Brasil | 2017

ISBN 978-85-212-1305-5
Proceedings of the $8^{\text {th }} \mathrm{CIDI}$ and $8^{\text {th }}$ CONGIC

Guilherme Santa Rosa; Cristina Portugal (orgs.)

Sociedade Brasileira de Design da Informação - SBDI

Natal| Brazil | 2017

ISBN 978-85-212-1305-5 


\section{CIDI 2017 先

Uma das áreas mais criticadas pelos docentes foi a listagem dos tópicos da disciplina. Na interface anterior, os tópicos eram disponibilizados de forma crescente, isto é, os itens mais recentes eram colocados abaixo daqueles mais antigos, em um único plano de fundo. A alternativa foi exibir tais informações destacando sempre os itens mais recentes ao topo. No redesign da interface, essa área informacional tornou-se um feed de atualizações da disciplina, remetendo a um mural, cujas informações sobre tópicos de disciplina, arquivos enviados pelo professor, entregas de atividades, dentre outros, foram organizadas e distribuídas em cards (variáveis de suporte), os quais poderiam receber comentários dos participantes e serem compartilhados via link (Figura 6). Assim, as atualizações funcionariam como postagens, em que os usuários pudessem interagir com mais facilidade com os assuntos da disciplina, além de buscar por conteúdos específicos (resolvendo a ausência do recurso de busca da interface anterior) ou filtrá-los por meio de palavras-chave (tags).

Figura 6: Atualizações da disciplina. (Elaboração dos autores)

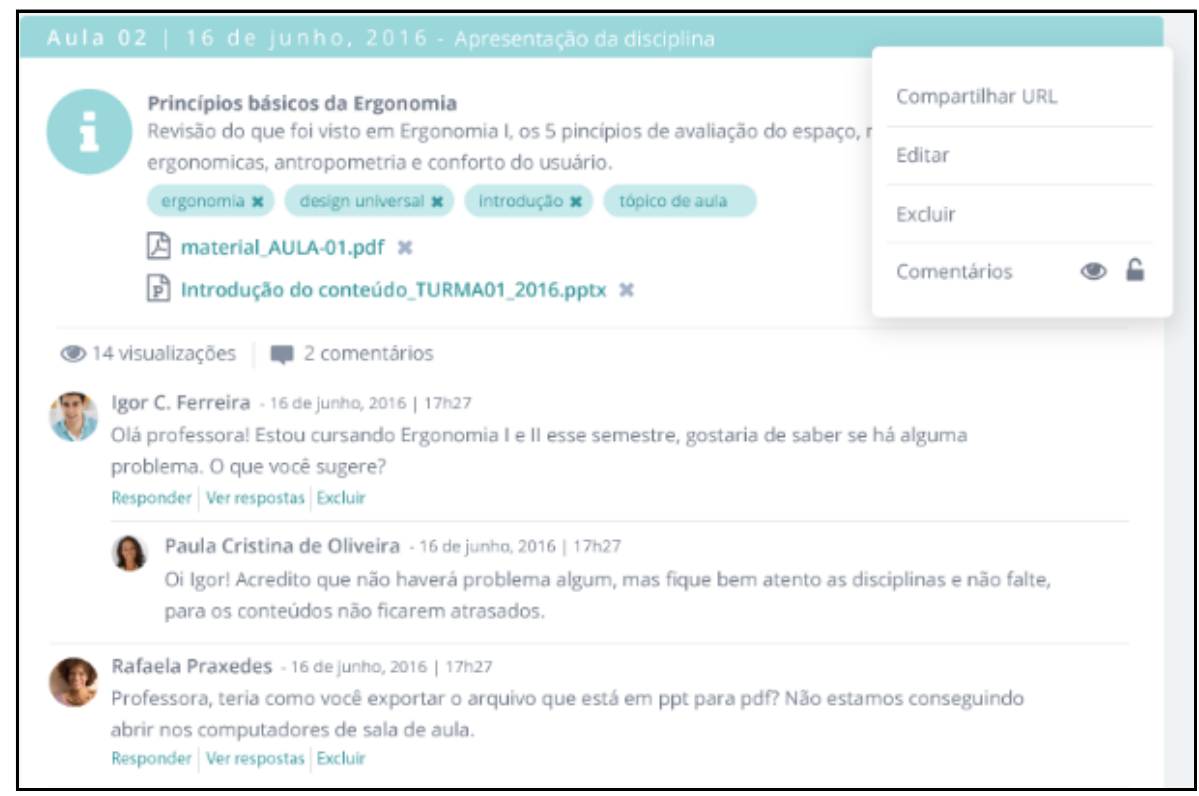

À direita, o docente tem acesso às opções que, em estudo anterior, foram identificadas como as funcionalidades frequentemente acessadas. Todas foram dispostas em cards, tornando a organização da interface marcada por variáveis de suporte. O usuário pode personalizar o menu, adicionando outras que Ihe sejam úteis (Figura 7).

Figura 7: Caixa de atalhos personalizável. (Elaboração dos autores)

Anais do $8^{\circ}$ CIDI e $8^{\circ}$ CONGIC

Guilherme Santa Rosa; Cristina Portugal (orgs.)

Sociedade Brasileira de Design da Informação - SBDI

Natal | Brasil | 2017

ISBN 978-85-212-1305-5
Proceedings of the $8^{\text {th }}$ CIDI and $8^{\text {th }}$ CONGIC

Guilherme Santa Rosa; Cristina Portugal (orgs.)

Sociedade Brasileira de Design da Informação - SBDI

Natal| Brazil | 2017

ISBN 978-85-212-1305-5 


\section{CIDI 2017 sel

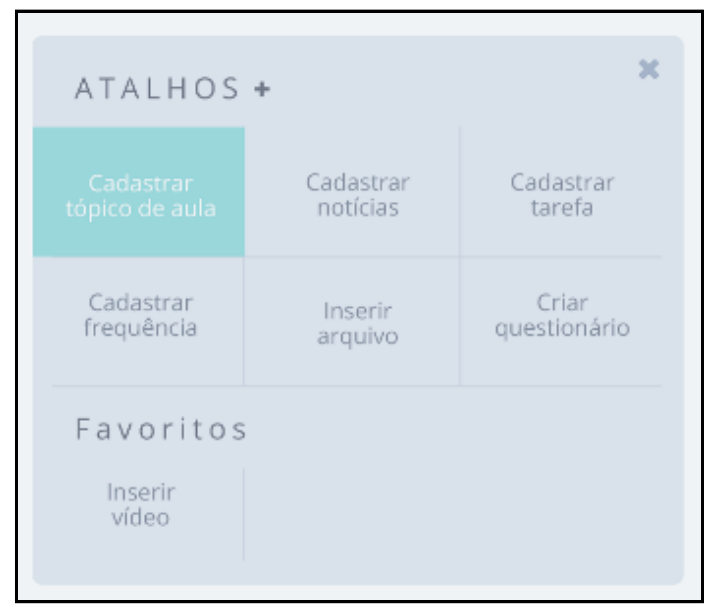

No calendário da turma, aplicaram-se cores como variáveis diferenciadoras para indicar atividades, avaliações e dias de aula, semelhante ao esquema de cores adotado no aplicativo móvel. (Figura 8).

Figura 8: Calendário da turma. (Elaboração dos autores)

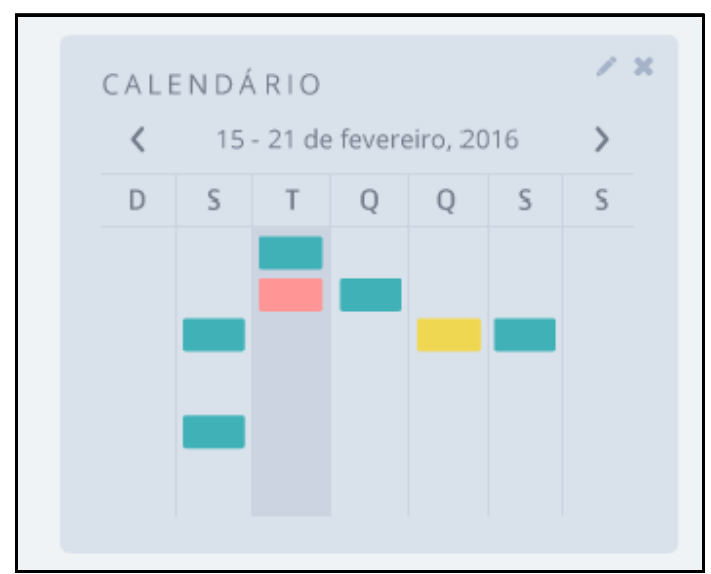

A Figura 9 exibe o cadastro de frequência, acentuadamente utilizado e de complexa organização, segundo os docentes. Aplicaram-se cores e ícones como variáveis diferenciadoras para as microinterações.

Figura 9: Cadastro de frequência. (Elaboração dos autores)

Anais do 8 CIDI e 8 CONGIC

Guilherme Santa Rosa; Cristina Portugal (orgs.)

Sociedade Brasileira de Design da Informação - SBDI

Natal | Brasil | 2017

ISBN 978-85-212-1305-5
Proceedings of the $8^{\text {th }} \mathrm{CIDI}$ and $8^{\text {th }}$ CONGIC

Guilherme Santa Rosa; Cristina Portugal (orgs.)

Sociedade Brasileira de Design da Informação - SBDI

Natal| Brazil | 2017

ISBN 978-85-212-1305-5 


\section{CIDI 2017 gin CIDI

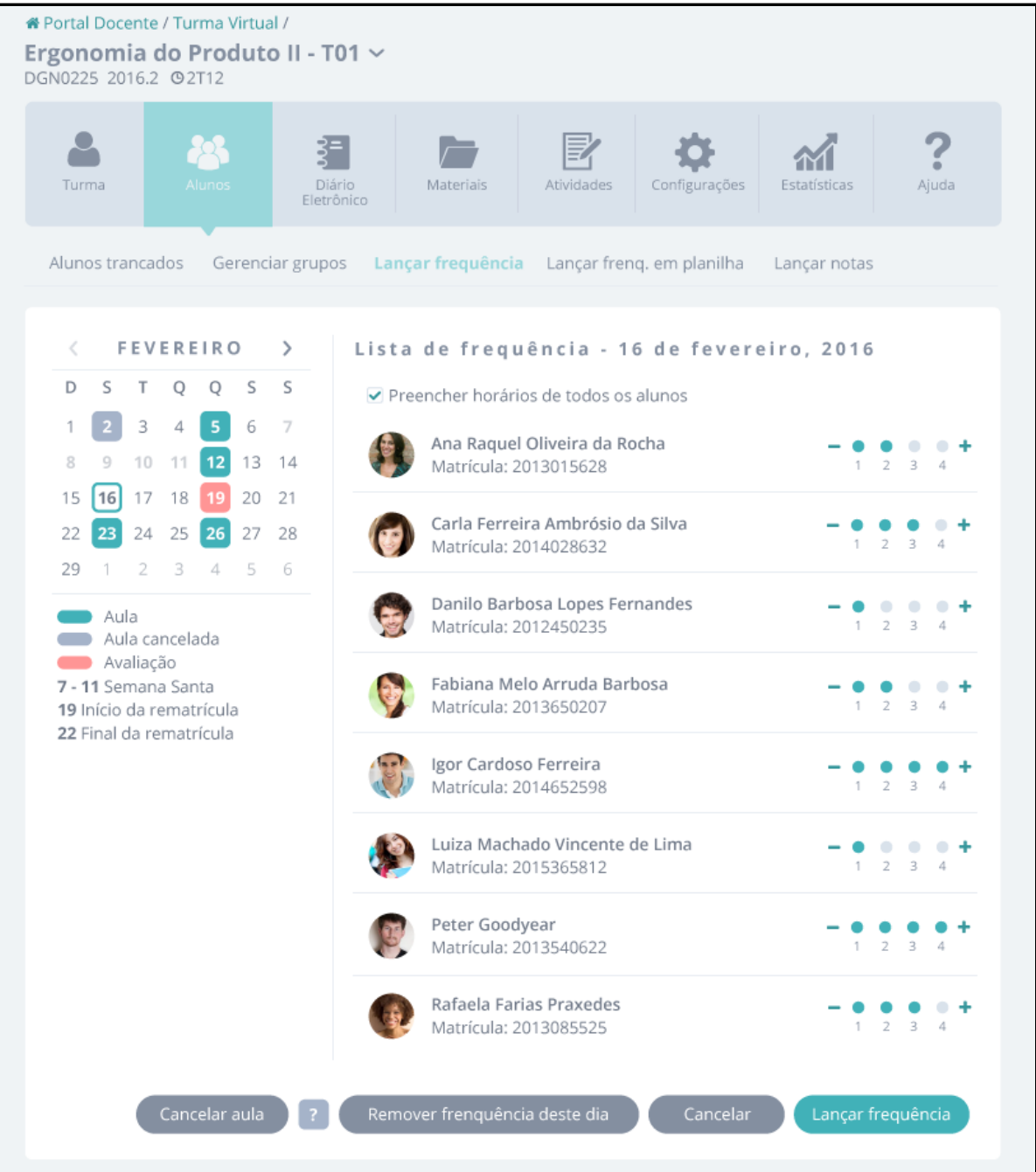

A acessibilidade foi discutida junto à equipe de desenvolvedores, sendo possível identificar abordagens de programação front-end que respaldassem a codificação da interface para navegações via teclado. Estudos realizados em conformidade com as diretrizes do eMAG e W3C (Brasil, 2014; W3C, 2016) fundamentaram versões em alto-contraste, para usuários com baixa visão. Neste caso, utilizou-se a cor como variável diferenciadora (Figura 10).

Figura 10: Interface em alto-contraste. (Elaboração dos autores)

Anais do 8 CIDI e 8 CONGIC

Guilherme Santa Rosa; Cristina Portugal (orgs.)

Sociedade Brasileira de Design da Informação - SBDI

Natal | Brasil | 2017

ISBN 978-85-212-1305-5
Proceedings of the $8^{\text {th }} \mathrm{CIDI}$ and $8^{\text {th }}$ CONGIC

Guilherme Santa Rosa; Cristina Portugal (orgs.)

Sociedade Brasileira de Design da Informação - SBDI

Natal| Brazil | 2017

ISBN 978-85-212-1305-5 


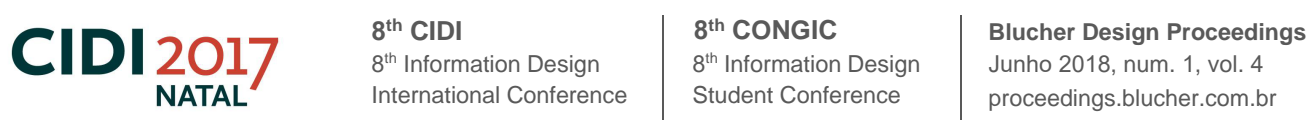

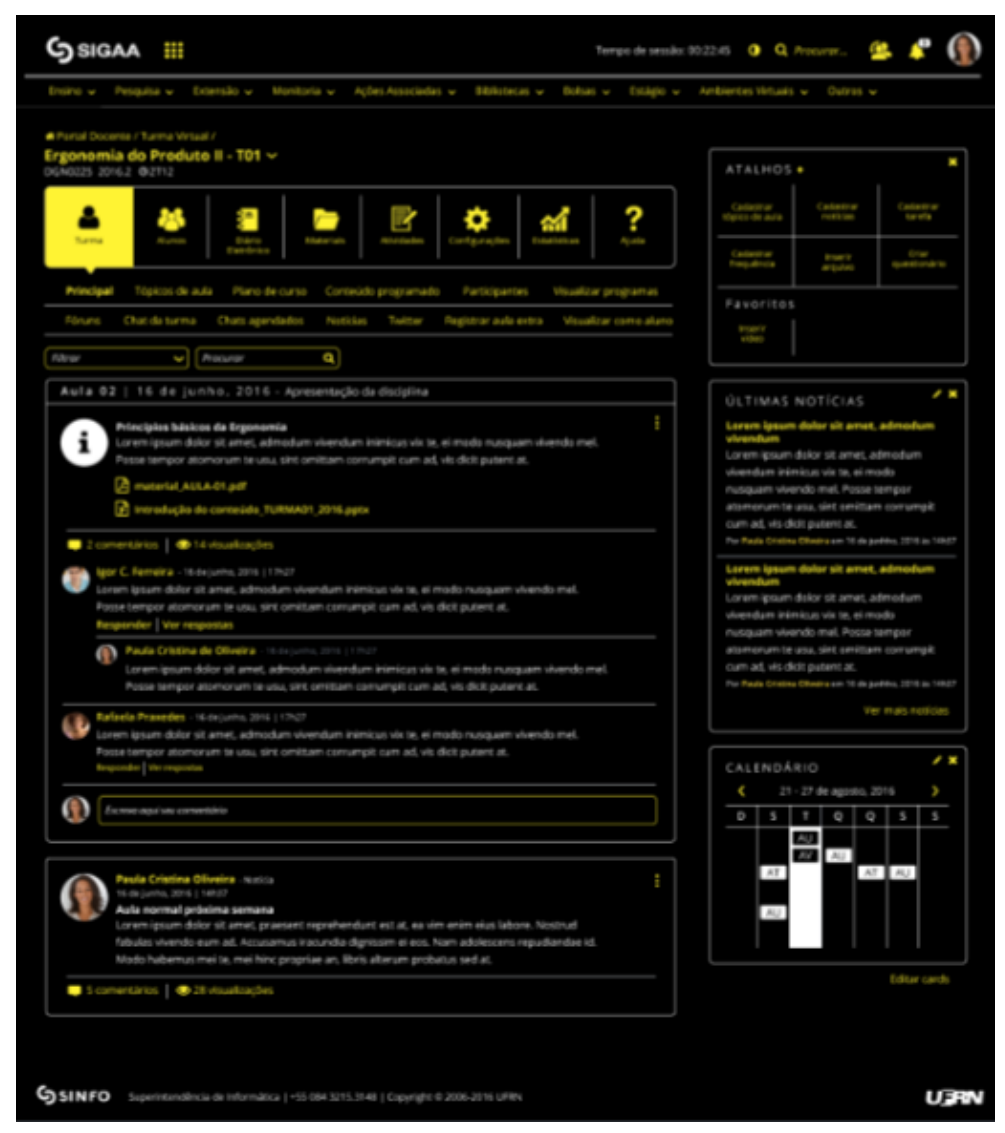

\subsection{Validação}

Participaram da validação da interface 103 docentes da UFRN, que responderam a um questionário fornecido junto à apresentação detalhada e em vídeo do protótipo. A faixa etária da maioria dos participantes (35,9\%) situou-se entre 31 e 40 anos de idade, seguidos de $29 \%$ entre 41 e 50 anos, $16 \%$ até 30 anos de idade e $15 \%$ entre 51 e 60 anos. Uma parcela de 2,9\% possuía mais de 60 anos de idade. Em relação ao tempo de atuação dos docentes, 35,9\% atuam na instituição numa média de 6 a 10 anos, 30,1\% entre 1 e 5 anos, $16,5 \%$ há mais de 20 anos, 9,7\% há menos de 1 ano, $4,9 \%$ de 16 a 20 anos e 2,9\% de 11 a 15 anos (Figura 11).

Figura 11: Tempo de atuação na Universidade. (Dados da pesquisa)

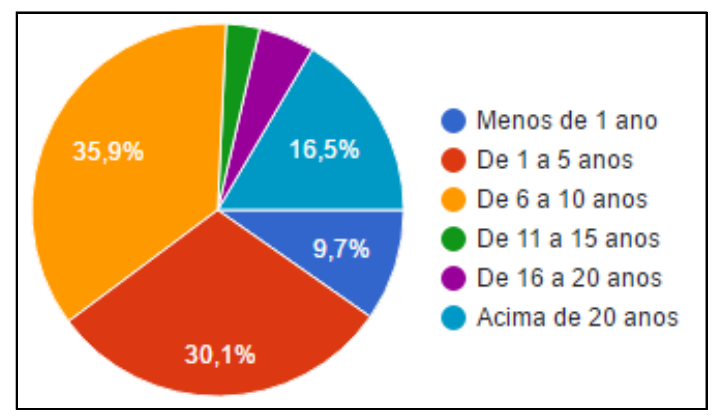

Em maioria, participaram docentes atuantes na graduação e na pós-graduação $(59,2 \%$ e $70,9 \%$, respectivamente). Uma parcela de $8,7 \%$ atua no ensino técnico oferecido na Universidade, e um dos participantes leciona em nível médio. Houve distribuição bastante variada dos locais de atuação de cada docente. A maioria das respostas se originaram do Centro de Tecnologia (19,4\%), Centro de Ciências Exatas e da Terra (13,6\%) e Centro de Ciências Humanas, Letras e Artes (10,7\%). Participaram docentes do

\section{Anais do 8 ㄷDI e 8 ㅇNGIC}

Guilherme Santa Rosa; Cristina Portugal (orgs.)

Sociedade Brasileira de Design da Informação - SBDI

Natal | Brasil | 2017

ISBN 978-85-212-1305-5
Proceedings of the $8^{\text {th }} \mathrm{CIDI}$ and $8^{\text {th }}$ CONGIC

Guilherme Santa Rosa; Cristina Portugal (orgs.)

Sociedade Brasileira de Design da Informação - SBDI

Natal| Brazil | 2017

ISBN 978-85-212-1305-5 


\section{CIDI 2017 ind

campus central, bem como dos interiores do Estado do Rio Grande do Norte, como do Centro de Ensino Superior do Seridó $(9,7 \%)$.

Dentre as áreas de conhecimento lecionadas pelos participantes, destacam-se os cursos: Sistemas de Informação, Filosofia, Pedagogia, Engenharia Mecânica, Gestão Hospitalar, História, Matemática, Ciências e Tecnologia, Letras, Engenharia de Software, Comunicação Social, Tecnologia da Informação, Direito, Fonoaudiologia, Medicina, Técnico em Informática, Ciências Biológicas, Turismo, Ciências Contábeis, Técnico em Agropecuária, Engenharia Elétrica, Engenharia de Telecomunicações, Arquitetura, Biomedicina, Gestão de Políticas Públicas, Enfermagem, Medicina, Ciências Atuariais, Engenharia do Petróleo, Química, Nutrição, Psicologia, Gestão Hospitalar, Física, Biblioteconomia, Administração, Ciências Econômicas, Geologia, Estatística, Geografia, Odontologia.

Quanto às opiniões sobre as ideias apresentadas, $66 \%$ dos docentes afirmaram estar totalmente satisfeitos com a página inicial, que exibe as atualizações na forma de feed; $25 \%$ responderam que estão parcialmente satisfeitos, 3,8\% insatisfeitos e 3,8\% são indiferentes. Quanto ao cadastro de frequência, $61 \%$ dos docentes estão totalmente satisfeitos, $31 \%$ responderam que estão parcialmente satisfeitos, $4,8 \%$ responderam que estão insatisfeitos e 2,9\% indicaram estar indiferentes. Para a criação de questionários, $56 \%$ docentes afirmaram estar totalmente satisfeitos, $28 \%$ estão parcialmente insatisfeitos, 1,9\% insatisfeitos e $13,59 \%$ responderam que são indiferentes. Quanto ao menu navegação, $70 \%$ dos docentes responderam que estão totalmente satisfeitos, $25 \%$ parcialmente satisfeitos, $2,9 \%$ indicaram estar insatisfeitos e 0,97\% afirmou estar indiferente (Figura 12).

Figura 12: Níveis de satisfação dos docentes com as soluções apresentadas. (Dados da pesquisa)

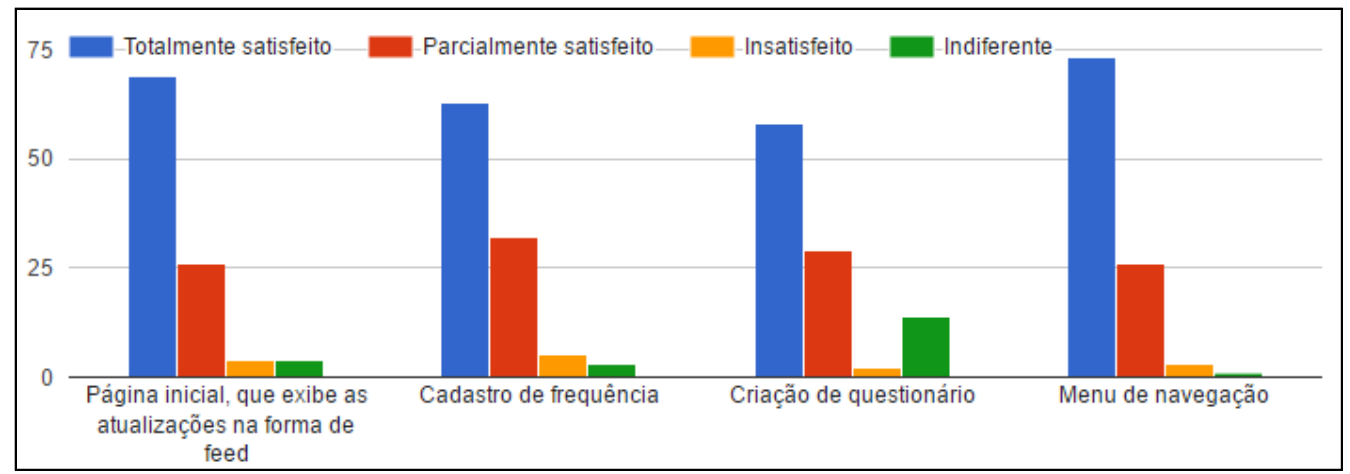

Foi solicitado aos respondentes que avaliassem o novo design apresentado (Tabela 2), utilizando uma escala de diferencial semântico de 0 (péssima) a 3 (ótima). A opinião dos docentes se demonstrou predominantemente receptiva às mudanças sugeridas com $57 \%$ considerando-as como ótimas (3). Nenhum dos participantes marcou a opção 0 (péssima).

Tabela 2: Avaliação geral dos participantes sobre a nova interface. (Dados da pesquisa)

\begin{tabular}{ccccc}
\hline & $\mathbf{0}$ & $\mathbf{1}$ & $\mathbf{2}$ & $\mathbf{3}$ \\
\hline Avaliação & Péssima & & & Ótima \\
\hline $\boldsymbol{f}(\%)$ & 0 & $3,9 \%$ & $38,8 \%$ & $57,3 \%$ \\
\hline
\end{tabular}

Foi disponibilizado um espaço no questionário para que os participantes pudessem registrar seus comentários e sugestões para o trabalho. Algumas das opiniões estão agrupadas no Quadro 1.

Anais do 8 CIDI e 8 CONGIC

Guilherme Santa Rosa; Cristina Portugal (orgs.)

Sociedade Brasileira de Design da Informação - SBDI

Natal | Brasil | 2017

ISBN 978-85-212-1305-5
Proceedings of the $8^{\text {th }} \mathrm{CIDI}$ and $8^{\text {th }}$ CONGIC

Guilherme Santa Rosa; Cristina Portugal (orgs.)

Sociedade Brasileira de Design da Informação - SBDI

Natal| Brazil | 2017

ISBN 978-85-212-1305-5 
"Gostaria de fazer uma sugestão dos alunos terem a possibilidade de inserir links ou conteúdo no campo em que o professor põe a aula. Não sei se isso teria uma aceitação por parte dos outros docentes, mas caso contrário, o professor poderia ter a opção de avaliar o conteúdo sugerido pelo aluno. Ficaria interativo, uma vez que o aluno também participa do conteúdo ofertado em sala. Parabéns!"

"Poderia agregar funções de acompanhamento acadêmico do estudante. Algumas informações que o docente poderia ter para um diagnóstico da turma. Algumas poderiam ser individualizadas e outras em forma de gráficos de histograma, pizza, barras, etc. Nesse caso cabe uma discussão devido à informações de cunho privado e público. Que permitam atividades diferenciadas e direcionadas."

"Além da organização em feeds tanto docentes precisam de uma opção para organizar e buscar o conteúdo. Isso pode ser feito, já na versão atual, inserindo nos posts do feed hashtags ou marcadores que seriam indexados em um menu da turma virtual. Por exemplo, eu poderia marcar um post como \#unidade1, \#atividade, \#android. Um outro como \#unidade1, \#webservices, etc. as tags ajudariam a formar um menu dinâmico (de acordo com sua repetição) e possibilitariam os discentes a buscar conteúdo de forma mais simples."

"Seria interessante existir a possibilidade de direcionar tarefas ou atividades isoladas, pois quando temos alunos afastados (atestado ou licença) temos que usar e-mail ou facebook que não são os canais de comunicação oficial da UFRN e ficamos obrigados a receber as atividades em outros canais de comunicação."

"Achei excelente a iniciativa, virou uma plataforma bem mais dinâmica e moderna."

"Gostei do visual, mais moderno. A uma primeira olhada, me pareceu mais amigável. Seria interessante fazer testes e avaliações utilizando uma versão beta de modo mais interativo, simulando uma situação real."
"Na versão atual os arquivos postados para uma turma ficam numa pasta chamada arquivo dentro de uma subpasta com o código da disciplina, porém para uma mesma disciplina os arquivos não ficam separados por semestre, ficam todos juntos para todos os semestres o que dificulta uma eventual correção. Sugiro que dentro da pasta com o código da disciplina seja criada uma nova pasta com o semestre correspondente."

"Parabéns pelo esforço. O sistema está cada vez melhor, responsivo, tornando-se uma ótima ferramenta de uso pedagógico também, e não apenas para preenchimentos burocráticos."

"Seria muito interessante não atrelar os materiais disponibilizados pelo professor em suas turmas virtuais à datas. Isso engessa muito a maneira como o material é disponibilizado, além de criar uma enorme dificuldade na hora de importar material em turmas futuras."

"Minha maior dificuldade é excluir um arquivo inserido no tópico de aula errado. Poderia ter um botão para excluir."

"Seria interessante que, em tarefas repetitivas, desse a possibilidade de não ter que sair da tela, para repetir a tarefa; - Em tarefas que possa haver a seleção de vários itens, que seja disponibilizada a possibilidade na tela de selecionar vários ou todos de uma vez."

"Uma das maiores dificuldades com o uso da turma virtual é de criar os tópicos de aula e poder manipular os tópicos de forma separada das datas. Quando cadastro 36 tópicos, e associo um a cada dia de aula, caso eu queria mudar um tópico de dia (adiantar) ou se atrasar uma aula, a atualização dos tópicos para as novas datas é bastante trabalhosa. Já que haverá essa nova forma (postagem), talvez isso possa ser melhorado também. A atualização das presenças ficou muito boa. Se puder ser feita alguma atualização na forma de cadastrar as notas, ajudaria também."

As opiniões dos docentes, com propriedade e experiência na prática diária com a ferramenta, oportunizaram novos encaminhamentos para a investigação, dentre os quais:

- Melhor gerenciamento de arquivos relacionados aos tópicos de aula e às disciplinas;

- Tornar a edição de tópicos de aula e cadastro de notas mais flexível;

- Implementar inteligência que sugira os dados inseridos com frequência pelo usuário, evitando ações repetitivas;

- Permitir que o professor gerencie quais postagens do feed podem receber comentários;

- Direcionar tarefas isoladamente para cada aluno se for necessário;

- Dispor menu com as principais tags utilizadas na turma, para facilitar as buscas rápidas.

\section{Conclusões e desdobramentos}

Foram investigados aspectos ergonômicos em interfaces de AVA na prática docente do ensino superior, buscando-se redução nos ruídos comunicacionais por meio critérios de design da informação, mediante modelos de análise. Neste esteio, o modelo de análise de variáveis visuais propostas por Mijksenaar (1997) satisfez o estudo da intervenção no objeto, bem como o apoio de um quadro visual para compor a visão

\section{Anais do 8 CIDI e 8 CONGIC}

Guilherme Santa Rosa; Cristina Portugal (orgs.)

Sociedade Brasileira de Design da Informação - SBDI

Natal | Brasil | 2017

ISBN 978-85-212-1305-5
Proceedings of the $8^{\text {th }} \mathrm{CIDI}$ and $8^{\text {th }}$ CONGIC

Guilherme Santa Rosa; Cristina Portugal (orgs.)

Sociedade Brasileira de Design da Informação - SBDI

Natal| Brazil | 2017

ISBN 978-85-212-1305-5 


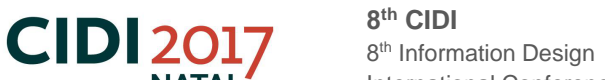

geral do problema permitiu compartilhamento de ideias com as partes envolvidas durante o processo.

Os resultados da avaliação do protótipo apontaram para satisfação dos docentes quanto às propostas para funcionalidades de uso mais frequente, bem como a organização dos elementos da interface gráfica. Houve predominante aprovação do novo arranjo do menu principal e também da inserção de um feed de atualizações da turma ( 66\% e $~ 70 \%$, respec., cf. Figura 12).

Os participantes manifestaram interesse em utilizar a ferramenta para avaliarem melhor seu desempenho e comportamento, solicitando a disponibilização de uma versão para testes, uma vez que o protótipo inicial foi demonstrado utilizando somente recursos audiovisuais. O objetivo da demonstração foi propor aos sujeitos um novo conceito de uso da ferramenta, para identificar oportunidades e obter direcionamentos para evolução do trabalho. Os feedbacks coletados serão utilizados no amadurecimento das alternativas apresentadas, sendo aquelas já validadas encaminhadas para o desenvolvimento de protótipos navegáveis, visando a oferecer uma versão funcional para validações in loco.

Dos parâmetros aplicados a esta investigação, conclui-se que:

- Os critérios de design da informação ora aplicados a este estudo, i.e., as variáveis visuais diferenciadoras, hierárquicas e de suporte -, atuam de maneira interdependente na composição gráfica da interface. A combinação entre estas permitiu realçar ou destacar elementos relevantes daqueles menos importantes. Verificou-se, ainda, a possibilidade de administrar o efeito das variáveis visuais nos esquemas cromáticos para acessibilidade, sendo tais critérios interessantes em abordagens inclusivas no design de interfaces;

- O emprego de ferramenta visual para organizar insights gerados no decorrer do projeto permitiu a socialização e ampliação do panorama da experiência dos usuários docentes, igualando o conhecimento do problema entre designers e desenvolvedores e propiciando gatilhos visuais para decisões colaborativas entre os profissionais da organização investigada. Tornou-se, assim, importante material de apoio para conscientização e consolidação de práticas de design em organizações de tecnologia da informação;

- Em um panorama sobre o objeto investigado e confrontando as questões levantadas inicialmente para a pesquisa, as intervenções na composição da interface, mediadas por critérios de design da informação, produziram efeitos positivos na percepção dos docentes usuários da ferramenta, vez que se observou na validação do protótipo por estes. Assim, aplicou-se o design da informação não apenas para diagnóstico ergonômico-cognitivo do artefato, mas como substrato teórico e técnico para seu melhoramento, de modo que o DI pode contribuir no projeto ergonômico-cognitivo da atividade de docentes do ensino superior em ambientes virtuais de aprendizagem.

Destaca-se a importância de considerar a voz dos usuários desde o início do processo de redesign da interface, uma vez que seus anseios, quando explicitados, tornaram-se dados qualitativos que direcionaram o processo e permitiram uma melhor visualização do problema, de forma que, além do olhar técnico em design sobre os elementos da interface, as opiniões dos usuários, carregadas de experiências e sentimentos vivenciados em seus cotidianos, trouxeram para o projeto novas questões ergonômicas.

Para próximas etapas, dar-se-á continuidade às abordagens participativas, com realização de avaliações cooperativas, dentre outras técnicas que possibilitem a construção de uma ferramenta centrada nos usuários e que promova benefícios para a experiência tecnológica dos docentes em suas metodologias de ensino.

\section{Agradecimento}

Os autores agradecem à Fundação Norte-Rio-Grandense de Pesquisa e Cultura (FUNPEC), pelo financiamento desta pesquisa; e à gestão da Superintendência de Informática da UFRN, por apoiar as ações de design na Instituição.

\section{Referências}

Agência de Comunicação da UFRN - AGECOM. Sinfo lança aplicativo Sigaa Mobile para sistema operacional iOS. In: Boletim Diário da UFRN, Natal-RN, terça-feira, 08 de março, no 43, Ano XV, 2016.

\section{Anais do 8 IDI e 8 CONGIC}

Guilherme Santa Rosa; Cristina Portugal (orgs.)

Sociedade Brasileira de Design da Informação - SBDI

Natal | Brasil | 2017

ISBN 978-85-212-1305-5
Proceedings of the $8^{\text {th }} \mathrm{CIDI}$ and $8^{\text {th }}$ CONGIC

Guilherme Santa Rosa; Cristina Portugal (orgs.)

Sociedade Brasileira de Design da Informação - SBDI

Natal| Brazil | 2017

ISBN 978-85-212-1305-5 


\section{CIDI 2017 ind

Associação Brasileira de Ergonomia - ABERGO. O que é Ergonomia. 2008. Disponível em: <http://www.abergo.org.br/internas.php?pg=o_que_e_ergonomia>. Acesso em 26 set 2016.

BERTIN, J. Sémiologie Graphique: Les diagrammes, les réseaux, les cartes. Paris: Gauthier-Villars, 1967.

BRASIL. Ministério do Planejamento, Orçamento e Gestão; Secretaria de Logística e Tecnologia da Informação. eMAG: Modelo de Acessibilidade em Governo Eletrônico. Versão 3.1, Abril/2014. Brasília: MP, SLTI, 2014.

CORTELLA, M.; \& DIMENSTEIN, G. A Era da Curadoria. Campinas-SP: Papirus 7 Mares, 2015.

GALAFASSI, F. P.; GLUZ, J. C.; \& GALAFASSI, C. Análise Crítica das Pesquisas Recentes sobre as Tecnologias de Objetos de Aprendizagem e Ambientes Virtuais de Aprendizagem. Revista Brasileira de Informática na Educação, [S.I.], v. 21, n. 03, p. 41, mar. 2014. ISSN 1414-5685. Disponível em: <http://www.br-ie.org/pub/index.php/rbie/article/view/2351/2457>. Acesso em: 25 set 2016.

GRILO, A. Adoção de práticas de design para inovação no desenvolvimento de sistemas acadêmicos: relato de experiência na Superintendência de Informática da UFRN. In: Anais do XXII Seminário de Pesquisa do CCSA, 08-12 maio. Natal: UFRN, 2017.

; SILVA, A. V.; COSTA, C. PRÁTICAS DE DESIGN E USABILIDADE NO DESENVOLVIMENTO DE SISTEMAS DE GESTÃO ACADÊMICA: ESTUDO DE CASO DE SISTEMA DE MEMORANDOS

ELETRÔNICOS NA UFRN. In: Anais do 16ํㅡㄴ Congresso de Ergonomia e Usabilidade em Interfaces HumanoComputador, 05-09 jul. Florianópolis-SC: 2017.

. Um Framework para Processos de Design para Web Considerando as Dimensões Semióticas do Produto. Revista Temática, João Pessoa, v. 12, n. 11, p. 31-43, nov/2016.

; DIAS, L. A Prática Docente e a Usabilidade em Ambientes Virtuais de Aprendizagem no Ensino Superior: Estudos na Turma Virtual do SIGAA. Revista Tecnologias na Educação, ano 8, n. 17, p. 70-83, dez/2016.

IIDA, I. Ergonomia: projeto e produção. 2 ed. São Paulo: Blucher, 2005.

Information Design Association - IDA. Origins. Disponível em: <http://www.infodesign.org.uk/About-theIDA/origins>. Acesso em 28 de maio de 2017.

International Institute for Information Design. About IIID. Disponível em: <http://www.iiid.net/home/aboutiiid/>. Acesso em 28 de maio 2017.

MIJKSENAAR, P. Visual Function: an Introduction to Information Design. Rotterdam: 010 Publishers, 1997. MORVILLE, P. \& ROSENFELD, L. Information Architecture for The World Wide Web. O'Reilly Media, Inc, 2007.

NIELSEN, J. 10 Usability Heuristics for User Interface Design. 1995. Disponível em: <https://www.nngroup.com/articles/ten-usability-heuristics/>. Acesso em 26 set 2016.

NORMAN, D. Design do futuro. Rio de Janeiro: Rocco, 2010.

NUNES, J. V.; \& GONÇALVES, B. S. Orientações para o Design de hipermídias para aprendizagem da língua espanhola na EaD. Revista Brasileira de Informática na Educação, [S.I.], v. 23, n. 01, 2015, p. 58-69. Disponível em: <http://www.br-ie.org/pub/index.php/rbie/article/view/2453/2847>. Acesso em: 25 set 2016.

OKADA, A. L. P. Desafio para EAD: como fazer emergir a colaboração e cooperação em ambientes virtuais de aprendizagem? In: SILVA, M. (org.). Educação Online. São Paulo: Loyola, 2003.

PEREIRA, A. T. C.; SCHMITT, V.; DIAS, M. R. A. Ambientes virtuais de aprendizagem. In: PEREIRA, A. C. (Org.). AVA: Ambientes Virtuais de Aprendizagem em diferentes contextos. Rio de Janeiro: Ciência Moderna, 2007, p. 2-22.

SAMARA, T. Guia de design editorial: manual prático para o design de publicações. Porto Alegre: Bookman, 2011.

SPIEKERMAN, E. A linguagem invisível da tipografia: escolher, combinar e expressar com tipos. São Paulo:

Anais do 8 CIDI e 8 CONGIC

Guilherme Santa Rosa; Cristina Portugal (orgs.)

Sociedade Brasileira de Design da Informação - SBDI

Natal | Brasil | 2017

ISBN 978-85-212-1305-5
Proceedings of the $8^{\text {th }} \mathrm{CIDI}$ and $8^{\text {th }}$ CONGIC

Guilherme Santa Rosa; Cristina Portugal (orgs.)

Sociedade Brasileira de Design da Informação - SBD

Natal| Brazil | 2017

ISBN 978-85-212-1305-5 


\section{CIDI $2017 \quad 8^{\text {th }}$ CIDI

Blucher, 2011.

Superintendência de Informática, Universidade Federal do Rio Grande do Norte - SINFO/UFRN. Disponível em: <https://docs.info.ufrn.br/doku.php>. Acesso em: jun. 2017.

Disponível em: <http://portalcooperacao.info.ufrn.br/pagina.php?a=sobre>. Acesso em: jun. 2017.

World Wide Web Consortium - W3C. Disponível em:

<https://www.w3.org/standards/webdesign/accessibility> e <https://www.w3.org/WAl/>. Acesso em jun 2016.

\section{Sobre os autores}

André Grilo, Mestre em Design, UFRN, Brasil <andregrilo@info.ufrn.br>

Thiago Marques, Mestre em Design, UFRN, Brasil <thiagomarques@info.ufrn.br> Laura Fernandes, Graduanda em Design, UFRN, Brasil <lauradias@info.ufrn.br>

G

a

b

r

i

e 